\title{
Refractory immune-mediated and haematological diseases: candidates for peptide receptor radiotherapy?
}

\author{
Dik J Kwekkeboom ${ }^{1}$, P Martin van Hagen ${ }^{2,3}$ and Eric P Krenning ${ }^{1,3}$ \\ Departments of ${ }^{1}$ Nuclear Medicine, ${ }^{2}$ Immunology and ${ }^{3}$ Internal Medicine, University Hospital Rotterdam, Rotterdam, The Netherlands \\ (Correspondence should be addressed to Dik J Kwekkeboom, Department of Nuclear Medicine, University Hospital Rotterdam, Dr Molewaterplein 40, \\ 3015 GD Rotterdam, The Netherlands; Email: djkwekkeboom@hotmail.com)
}

\section{Introduction}

Somatostatin receptors have been demonstrated in the brain, in the pituitary gland, and on endocrine pancreatic islet cells $(1,2)$. These receptors may also be expressed on mostly neuroendocrine tumours (3) and in areas infested by certain immune-mediated diseases (4-7). The presence or overexpression of somatostatin receptors on these tumours and in these diseases is used when in vivo somatostatin receptor imaging (SRS) is performed in patients. A chelated, radiolabelled somatostatin analogue, $\left[{ }^{111}{ }^{2 n-D T P A}{ }^{0}\right]$ octreotide (OctreoScan, Mallinckrodt, Petten, the Netherlands), was injected and images were made after an interval of usually $24 \mathrm{~h}$, which allows for primarily urinary and also, to a very small extent, hepatobiliary excretion. By then, background radioactivity had dropped considerably. Normal scintigraphic features due to receptor binding include visualization of the pituitary (to a variable degree), the thyroid and the spleen, whereas visualization of the liver, kidneys and urinary bladder mainly represents the metabolization of the radiopharmaceutical. Because of the normal accumulation of radioactivity in the above-mentioned organs, but especially in the spleen, kidneys, and to a lesser degree in the liver, the detection of somatostatin receptor-positive pathology in or near these organs may be hampered. This may be overcome by performing single photon energy computed tomography but, in diseases with low receptor density, the detection of lesions in the upper abdomen may be difficult. If receptor density is high, however, the recognition of lesions usually poses no problems. Therefore, in neuroendocrine gastroenteropancreatic (GEP) tumours, which have a high receptor density, SRS has been found to be more sensitive than other imaging techniques, and is advocated as the imaging technique of choice in the staging procedure (8).

SRS may also demonstrate lesions in a number of non-neuroendocrine tumours, such as, for instance, breast carcinoma, Hodgkin's disease and non-Hodgkin lymphoma (NHL), and in immune-mediated diseases like rheumatoid arthritis and granulomatous diseases. However, its role in the diagnosis of these diseases is very limited, and its value in staging and monitoring the response to therapy remains to be evaluated.

\section{Localization of somatostatin receptors in haematological and immune-mediated disease and results of SRS}

Somatostatin receptors have been demonstrated by autoradiography with $\left[{ }^{125} \mathrm{I}-\mathrm{Tyr}^{3}\right]$-octreotide on granuloma tissue (5). The receptors were located in the areas containing epitheloid cells and not in the zone of surrounding lymphocytes. However, a more precise identification of the cells expressing somatostatin receptors, using receptor antibodies, showed that macrophages and epitheloid cells are the targets for receptor binding in granulomatous diseases (6). It is also of interest that biopsies that were negative for somatostatin receptor staining were characterized by fibrosis and the absence of an active monoclear infiltrate, indicating that receptor expression may be correlated to active disease. Using in vivo SRS in a cross-sectional study in 46 patients with sarcoidosis, known mediastinal, hilar and interstitial disease was recognized in 36 of 37 patients (9). Also, such pathology was found in seven other patients who had normal chest X-rays. In five of these, SRS indicated interstitial disease. SRS was repeated in 13 patients. In five of six patients who showed a chest X-ray-monitored improvement in disease activity, SRS also showed a decrease in pathologic uptake. In two of five patients in whom the chest X-ray was unchanged, but serum angiotensin converting enzyme concentrations were decreased and lung function was improved, a normalization was found on octreotide scintigrams. To determine the value of SRS in the follow-up of patients with sarcoidosis a prospective longitudinal study will have to be performed.

In the synovial membranes of patients with rheumatoid arthritis, high affinity binding of $\left[{ }^{125}{\left.\mathrm{I}-T y r^{3}\right] \text {-octreotide has }}\right.$ been shown (4). With receptor antibodies, staining for somatostatin subtype 2 receptors was found on a macrophage subset (7). In vivo, $76 \%$ of disease-affected joints in patients with rheumatoid arthritis were visualized with SRS (Figs 1 and 2). The degree of pain and swelling correlated with positive scintigraphic findings (4).

With autoradiography, somatostatin receptors have been demonstrated on human lymphomas (10-12). Although in many patients with NHL one or more lesion may be somatostatin receptor-positive (Fig. 3), receptornegative lesions also occur in a substantial number of patients (11). Therefore, the diagnostic role for SRS in patients with NHL is limited. In 56 consecutive untreated 


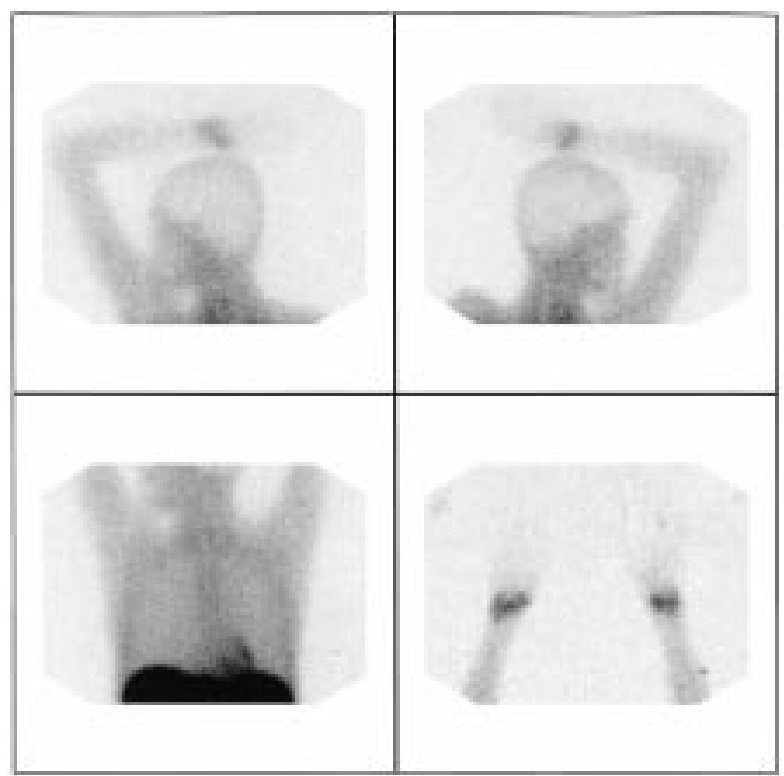

Figure $1 \mathrm{SRS}$ in a patient with arthritis, $24 \mathrm{~h}$ post-injection. Lateral images of the head (upper row), and anterior view of the chest, and detail of the wrists and hands (lower row). Pathological uptake in both wrists and also in the myocardium are shown.

patients with histologically proven Hodgkin's disease the results of SRS were compared with physical and radiological examinations (12). SRS was positive in 55 of $56(98 \%)$ patients at the sites of documented disease. In 20 patients, SRS disclosed lymphoma localizations not

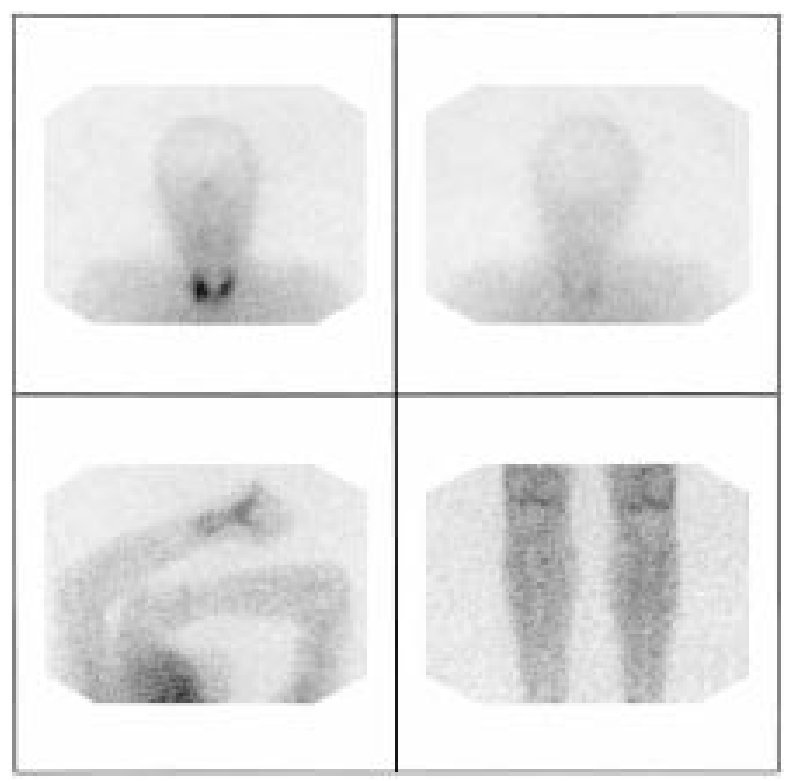

Figure 2 SRS in a patient with arthritis, $24 \mathrm{~h}$ post-injection. Anterior and posterior views of the head (upper row), and upper and lower extremities (lower row). Normal uptake in the pituitary and thyroid (upper left image) and pathological uptake in the right wrist (lower left image) and both knees (lower right image) are shown. revealed following procedures for conventional staging. As a result, in 12 patients (21\%), SRS produced a change of stage and in seven patients $(13 \%)$ the additional information led to a change of treatment. Therefore, SRS seems to be promising in the clinical staging and management of patients with Hodgkin's disease.

\section{Peptide receptor radionuclide therapy (PRRT), a sequel to SRS}

In patients with end-stage progressive neuroendocrine tumours which, in the majority, showed a high uptake of $\left[{ }^{111} \mathrm{In}-\mathrm{DTPA}{ }^{0}\right]$-octreotide during in vivo SRS, radionuclide therapy with high doses of $\left[{ }^{111} \mathrm{In}-\mathrm{DTPA}{ }^{0}\right]$ octreotide was performed in a phase-1 study. In 30 patients who received up to a cumulative dose of $74 \mathrm{GBq}$, the side-effects were a (transient) decline in platelet counts and lymphocyte subsets. Of the 21 patients who received a cumulative dose of more than $20 \mathrm{GBq}$, a reduction in tumour size was found in six, and stable disease in eight. There was a tendency towards better results in patients with a high tumour uptake (13).

${ }^{111}$ In-coupled peptides, because of their small particle range, and therefore short tissue penetration, are not the ideal radiopharmaceuticals for radionuclide therapy. Recently, another somatostatin analogue, $\left[\right.$ DOTA $^{0}$, Tyr $\left.^{3}\right]$-octreotide (DOTATOC), has been developed, to which the $\beta$-emitter ${ }^{90} \mathrm{Y}$ can be linked in a very stable manner (14). In a recent study (15), the uptake of radioactivity in known somatostatin receptor-positive organs and tumours was higher after ${ }^{11}$ InDOTATOC than after $\left[{ }^{111}\right.$ In-DTPA $\left.{ }^{0}\right]$-octreotide. A recent preliminary study by Otte et al. (16) showed favourable results of ${ }^{90}$ Y-DOTATOC treatment in five patients with neuroendocrine tumours. Also, a study has been started in our hospital comparing both the uptake of ${ }^{111}$ In-DOTATOC and ${ }^{86}$ Y-DOTATOC, as well as the effects of treatment with ${ }^{90}$ Y-DOTATOC in the same patients. Because the kidney is the dose-limiting organ in PRRT with ${ }^{90}$ Y-DOTATOC, an infusion of amino acids is given during and after the infusion of the radiopharmaceutical, in order to reduce the kidney uptake. A recent analysis of the results of this treatment in 22 end-stage patients with progressive disease shows a partial tumour response in two, a minor response in three, and stable disease in ten patients (17).

Because of favourable results in terms of tumour regression in animals after therapy with another somatostatin analogue, $\left[{ }^{177} \mathrm{Lu}-\mathrm{DOTA}, \mathrm{Tyr}^{3}\right]$-octreotate (18), the first injections of $1850 \mathrm{MBq}$ of this radiopharmaceutical were very recently given to six patients in our hospital. A preliminary comparison with $\left[{ }^{111} \mathrm{In}\right.$ $\mathrm{DTPA}^{0}$ ]-octreotide points to a higher tumour uptake, whereas the kidney uptake for both radiopharmaceuticals is the same. This implies that the radiotherapeutical dose to the tumour potentially increases with ${ }^{[177} \mathrm{Lu}$-DOTA,Tyr $\left.{ }^{3}\right]$-octreotate, given a fixed maximum kidney dose. In addition, the application of 


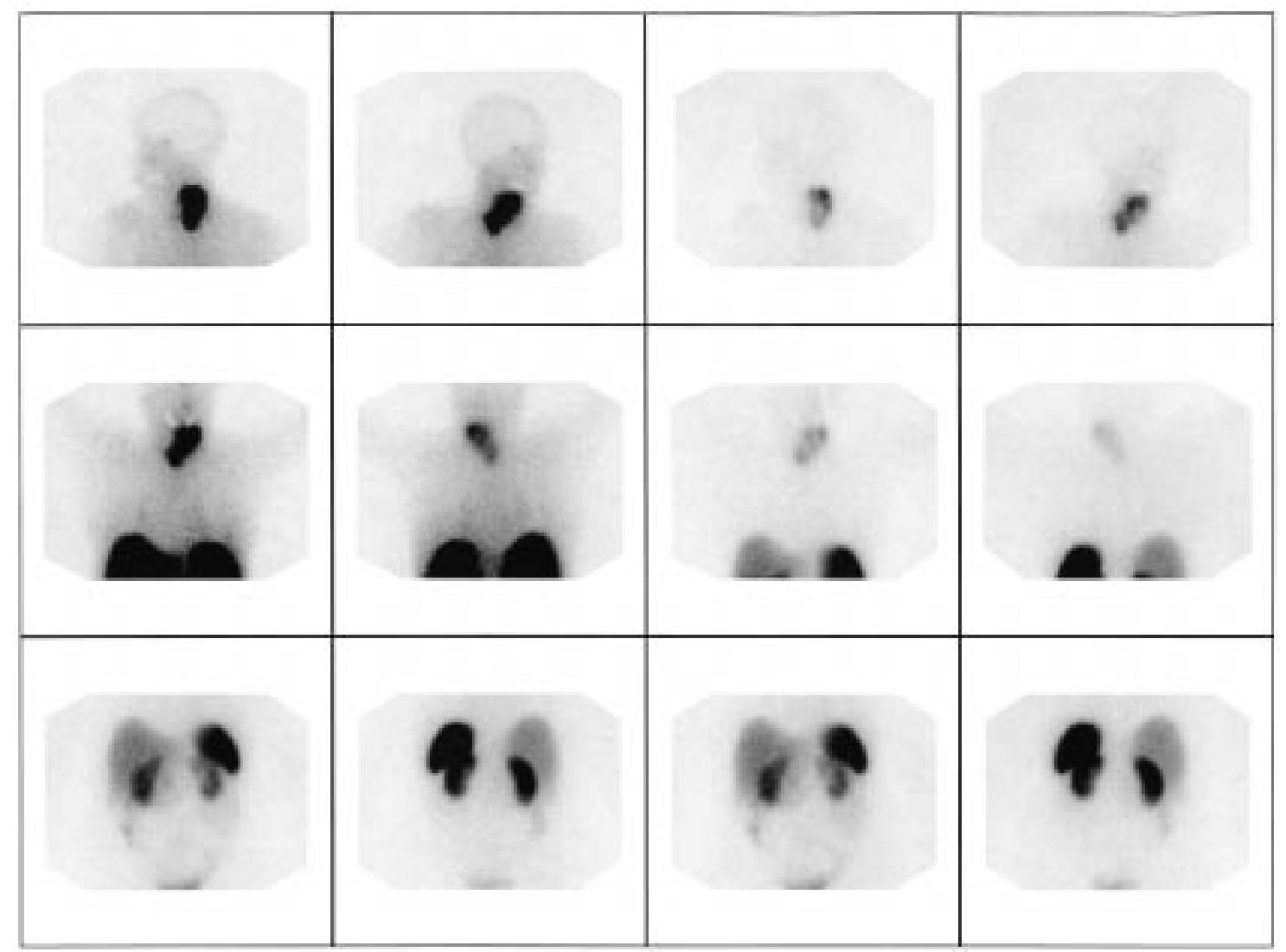

Figure 3 SRS in a patient with NHL, $24 \mathrm{~h}$ post-injection. Lateral views of the head (upper row) with normal uptake in the pituitary and pathological uptake at the site of a lymphoma in the left neck are shown. Anterior and posterior view of the chest (middle row), showing that the lymphoma uptake is comparable to (normal) uptake in the liver (bottom row). Normal, intense uptake in the spleen can also be seen (bottom row). The left two columns are scaled to allow for good visualization of the lymphoma. The right two columns are scaled to allow for comparison of the uptake in the lymphoma and in the liver.

$\left[{ }^{177} \mathrm{Lu}-\mathrm{DOTA}, \mathrm{Tyr}^{3}\right]$-octreotate, which emits both gamma and beta rays, allows dosimetry and therapy with the same radiopharmaceutical.

\section{Is PRRT a treatment option in patients with haematological or immune- mediated diseases?}

Combination chemotherapy, external beam radiotherapy and bone marrow transplantation, as single modality or in combination, are the corner-stones of treatment for Hodgkin's disease and NHL. High response and cure rates are achieved, and the results of changing the therapeutic strategies keep improving (19). As in patients with neuroendocrine tumours, PRRT with radiolabelled somatostatin analogues might be applied in patients with Hodgkin's disease or NHL if the tumour uptake is sufficient. In patients with neuroendocrine tumours, the results of PRRT are better if the tumour uptake equals the uptake in the liver or is greater. In patients with lymphomas, however, the majority have a lower tumour uptake. In addition, it should be realized that lymphoma patients who have shown to be refractory to standard and salvage chemotherapy and radiotherapy may very well have developed bone marrow and renal toxicity, which are also the two most important potential side-effects of PRRT. Also, as a rule, little benefit can be expected from PRRT if previous external beam radiotherapy in these patients did not affect tumour growth. Finally, in most patients with therapy-refractory lymphomas disease progression is fast; with the present treatment schemes of PRRT, the dose delivery to the tumour may therefore be too slow. Despite all these arguments against its use, PRRT could be tried in some patients who fail to respond to 'standard' therapy on the condition that there is a high tumour uptake.

Therapy in patients with sarcoidosis is symptomatic. Because of the high rate of spontaneous remission in patients with limited disease stages, corticosteroid treatment is best reserved for patients with active pulmonary sarcoidosis or patients with pulmonary function impairment, dyspnea, cough, chest pain, hypercalcaemia or extrapulmonary organ involvement 
$(20,21)$. Because somatostatin subtype 2 a receptors in granulomas were found on macrophages and epithelioid cells (6) and on a macrophage subset in the synovium from patients with rheumatoid arthritis (7), PRRT with radiolabelled somatostatin analogues, if effective, will probably affect these constituents of the inflammation. In vitro, the inhibition of cytokine release from monocytes (22) and diminished granuloma size in a mouse schistosomiasis model (23) by octreotide were demonstrated. Whether PRRT may lead to a decrease of the inflammatory response and of granuloma size in patients is, at this moment, speculative.

In patients with refractory rheumatoid arthritis, so-called 'radiosynoviorthesis' or 'radiosynovectomy' with $\beta$-emitting radionuclides (mostly ${ }^{90} \mathrm{Y}$ ) may cause symptomatic relief in the treated joints $(24,25)$. Usually, depending on the size of the joints to be treated, 37$185 \mathrm{MBq}$ are injected intra-articularly, resulting in up to about $100 \mathrm{~Gy}$ (10 000 Rad) radiation-absorbed dose. Because only a limited number of joints can be treated with this method, the advantage of PRRT, resulting in radiation to all joints that show uptake, seems evident. The degree of uptake of radioactivity during SRS in rheumatoid joints is usually low, however, as is usually the case in granulomatous lesions. It may therefore be questioned whether a sufficient dose can be delivered. On the other hand, if PRRT selectively affects the cells that are responsible for the immune response, such lower doses could be sufficient.

As mentioned previously, PRRT may cause bone marrow and renal toxicity. Such side-effects will have to be weighed against the possible benefits of treatment. PRRT should therefore, in our opinion, only be tried in patients with invalidating, progressive granulomatous disease or rheumatoid arthritis who are refractory to other treatments.

\section{References}

1 Patel YC, Amherdt M \& Orci L. Quantitative electron microscopic autoradiography of insulin, glucagon and somatostatin binding sites on islets. Science $1982 \mathbf{2 1 7} 1155-1156$.

2 Reubi JC \& Maurer R. Autoradiographic mapping of somatostatin receptors in the rat CNS and pituitary. Neuroscience 198515 1183-1193.

3 Reubi JC, Kvols LK, Waser B, Nagorney DM, Heitz PU, Charboneau JW et al. Detection of somatostatin receptors in surgical and percutaneous needle biopsy samples of carcinoids and islet cell carcinomas. Cancer Research 199050 5969-5977.

4 van Hagen PM, Markusse HM, Lamberts SWJ, Kwekkeboom DJ, Reubi JC \& Krenning EP. Somatostatin receptor imaging: the presence of somatostatin receptors in rheumatoid arthritis. Arthritis and Rheumatism 199437 1521-1527.

5 van Hagen PM, Krenning EP, Reubi JC, Kwekkeboom DJ, Bakker $\mathrm{WH}$, Mulder $\mathrm{AH}$ et al. Somatostatin analogue scintigraphy in granulomatous diseases. European Journal of Nuclear Medicine 199421 497-502.

6 Ten Bokum AMC, Hofland LJ, de Jong G, Bouma J, Melief MJ, Kwekkeboom DJ et al. Immunohistochemical localization of somatostatin receptor $\mathrm{SST}_{2 \mathrm{~A}}$ in sarcoid granulomas. European Journal of Clinical Investigation 199929 630-636.
7 Ten Bokum AMC, Melief MJ, Schonbrunn A, van der Ham F, Lindeman J, Hofland LJ et al. Immunohistochemical localization of somatostatin receptor $\mathrm{sst}_{2 \mathrm{~A}}$ in human rheumatoid synovium. Journal of Rheumatology $199926532-535$.

8 Gibril F, Reynolds JC, Doppman JL, Chen CC, Venzon DJ, Termanini B et al. Somatostatin receptor scintigraphy: its sensitivity compared with that of other imaging methods in detecting primary and metastatic gastrinomas. A prospective study. Annals of Internal Medicine 1996125 26-34.

9 Kwekkeboom DJ, Krenning EP, Kho GS, Breeman WAP \& Van Hagen PM. Octreotide scintigraphy in patients with sarcoidosis. European Journal of Nuclear Medicine 199825 1284-1292.

10 Reubi JC, Waser B, van Hagen PM, Lamberts SWJ, Krenning EP, Gebbers JO et al. In vitro and in vivo detection of somatostatin receptors in human malignant lymphomas. International Journal of Cancer $1992 \mathbf{5 0} 895-900$.

11 Van Hagen PM, Krenning EP, Reubi JC, Mulder AH, Bakker WH, Oei HY et al. Somatostatin analogue scintigraphy of malignant lymphomas. British Journal of Haematology 199383 75-79.

12 Van den Anker-Lugtenburg PJ, Krenning EP, Oei HY, van Hagen PM, Gerrits CJH, Reubi JC et al. Somatostatin receptor scintigraphy in the initial staging of Hodgkin's disease. British Journal of Haematology 199693 96-103.

13 Krenning EP, De Jong M, Kooij PPM, Breeman WA, Bakker WH, de Herder WW et al. Radiolabelled somatostatin analogue(s) for peptide receptor scintigraphy and radionuclide therapy. Annals of Oncology 199910 23-29.

14 Otte A, Jermann E, Behe M, Goetze M, Bucher HC, Roser HW et al. DOTATOC: a powerful new tool for receptor-mediated radionuclide therapy. European Journal of Nuclear Medicine 199724 $792-795$.

15 Kwekkeboom DJ, Kooij PPM, Bakker WH, Mäcke HR \& Krenning EP. Comparison of Indium-111-DOTATOC and Indium-111-DTPAoctreotide in the same patients: biodistribution, kinetics, organ and tumor uptake. Journal of Nuclear Medicine 199940 762-767.

16 Otte A, Mueller-Brand J, Dellas S, Nitzsche EU, Herrmann R \& Maecke HR. Yttrium-90-labelled somatostatin-analogue for cancer treatment. Lancet 1998351 417-418.

17 Valkema R, Jamar F, Jonard P, Bakker WH, Norenberg J, Hadley J et al. Targeted radiotherapy with ${ }^{90}$ Y-SMT487 (OctreoTher): a phase I study. Journal of Nuclear Medicine 200091 (Suppl.) 111P.

18 Erion JL, Bugaj JE, Schmidt MA, Wilhelm RR \& Srinivasan A. High radiotherapeutic efficacy of [Lu-177]-DOTA-Y ${ }^{3}$-octreotate in a rat tumor model. Journal of Nuclear Medicine $199940223 \mathrm{P}$.

19 DeVita VT Jr \& Canellos GP. The lymphomas. Seminars in Hematology $19993684-94$.

20 Saboor SA \& Johnson NMcI. Sarcoidosis. British Journal of Hospital Medicine 199248 293-302.

21 Weissler JC. Southwestern Internal Medicine Conference Sarcoidosis: immunology and clinical management. American Journal of the Medical Sciences 1994307 233-245.

22 Peluso G, Petillo O, Melone MAB, Mazzarella G, Ranieri M \& Tajana GF. Modulation of cytokine production in activated human monocytes by somatostatin. Neuropeptides 199630 443-451.

23 Elliott DE \& Weinstock JV. Granulomas in murine Schistosomiasis mansoni have a somatostatin immunoregulatory circuit. Metabolism $1996 \mathbf{8} 88-90$.

24 O'Duffy EK, Clunie GP, Lui D, Edwards JC \& Ell PJ. Double blind glucocorticoid controlled trial of samarium-153 particulate hydroxyapatite radiation synovectomy for chronic knee synovitis. Annals of the Rheumatic Diseases 199958 554-558.

25 Gratz S, Gobel D, Behr TM, Herrmann A \& Becker W. Correlation between radiation dose, synovial thickness, and efficacy of radiosynoviorthesis. Journal of Rheumatology $1999261242-$ 1249 .

Received 1 July 2000

Accepted 19 July 2000 